\title{
UM ESTUDO COMPARATIVO SOBRE A IDENTIFICAÇÃO DOS RISCOS \\ OCUPACIONAIS POR TRABALHADORES DE ENFERMAGEM DE DUAS UNIDADES BÁSICAS DE SAÚDE DO MUNICIPIO DE SAO PAULO
}

\section{A COMPARATIVE STUDY ABOUT IDENTIFICATION OF OCCUPATIONAL RISKS BY NURSING WORKERS OF TWO BASIC HEALTH UNITS OF SAO PAULO}

\section{UN ESTUDIO COMPARATIVO SOBRE LA IDENTIFICACIÓN DE LOS RIESGOS OCUPACIONALES POR TRABAJADORES DE ENFERMERIA DE DUAS UNIDADES BASICAS DE SALUD DEL MUNICIPIO DE SAO PAULO}

Rita de Cassia Gengo e Silva* Vanda Elisa Andres Felli**

Silva RCG, Felli VEA. Um estudo comparativo sobre a identificação dos riscos ocupacionais por trabalhadores de enfermagem de duas Unidades Básicas de Saúde do município de São Paulo. Rev Esc Enferm USP 2002; 36(1): 18-24.

\begin{abstract}
RESUMO
Os riscos ocupacionais do trabalho de enfermagem em Unidades Básicas de Saúde (UBS) não têm sido frequentemente abordados, o que nos estimulou a realizar este estudo com os objetivos de comparar a percepção dos trabalhadores de enfermagem de duas UBS sobre esses riscos e estabelecer um paralelo sobre os problemas de saúde relacionados com a sua exposição. Os dados foram coletados através da entrevista e analisados segundo suas frequências relativa e absoluta e coeficientes de risco. Os maiores coeficientes de risco estão relacionados a exposição aos riscos biológicos. Conclui-se que os trabalhadores têm dificuldade em apreender a gênese dos riscos ocupacionais.
\end{abstract}

PALAVRAS-CHAVE: Saúde ocupacional. Riscos ocupacionais- Enfermagem.

\begin{abstract}
Occupational risks of nursing work in Basic Health Units have not been study frequently, so it stimulate us to develop this paper, whose objective are: to compare the perception of nursing workers of two Basic Health Units about these risks and to establish a parallel between the health problems with their exposition to occupational risks- Data were collected by an interview and were analyzed according to their frequencies. Biological risks were observed more frequently. The conclusions show that nursing workers have difficult to understand the genesis of occupational risks.
\end{abstract}

KEYWORDS: Occupational health. Occupational risks. Nursing.

\section{RESUMEN}

Los riesgos ocupacionales del trabajo de enfermeria en Unidades Básicas de Salud (UBS) no han sido frecuentemente abordados, lo que nos estimuló en realizar este estudio con el objetivo de comparar la percepción de los trabajadores de enfermeria de dos UBS sobre estos riesgos y establecer un paralelo sobre los problemas de salud relacionados con su exposición. Los datos fueron recolectados a través de una entrevista y analizados según sus frecuencias relativa, absoluta y coeficientes de riesgo. Los mayores coeficientes de riesgo estan relacionados a la exposición de riesgos biológicos. Se concluye que los trabaj adores tienen dificultades en aprender la génesis de los riesgos ocupacionales.

PALABRAS-CLAVE: Salud ocupacional. Riesgos laborales. Enfermeria.

\footnotetext{
* Aluna do $8^{\circ}$ semestre do curso de graduação da Escola de Enfermagem da Universidade de São Paulo

** Enfermeira. Professora Doutora junto ao Departamento de Orientação Profissional da Escola de Enfermagem da USP.
} 


\section{INTRODUÇÃO}

Os ambientes de trabalho em que atuam os profissionais de enfermagem, por sua natureza, concentram uma série de riscos que podem trazer diversos problemas de saúde aos profissionais que nele trabalham, especialmente aos trabalhadores de enfermagem.

Esses problemas são gerados pela exposição dos trabalhadores aos riscos ocupacionais, entendidos como agentes nocivos isolados que são capazes de causar doença(1).

Silva (2) constrói um perfil de morbidade dos trabalhadores de enfermagem onde se destacam: ferimentos pérfuro-cortantes, doenças do aparelho ósteo-músculo-articular, doenças infecciosas ou infecto-contagiosas e parasitárias.

Laurell(1), ao sistematizar a relação trabalhosaúde, conceituam as cargas de trabalho, entendendo que estas abarcam os riscos ocupacionais, segundo a materialidade que assumem em relação ao corpo do trabalhador. Assim, as cargas podem ser de materialidade externa (independem do corpo do trabalhador) ou interna (quando só podem ser identificadas através do corpo do trabalhador). Silva(2), considerando o conceito de cargas de trabalho segundo Laurel1(1), as classifica da seguinte maneira para o trabalho de enfermagem:

- Cargas biológicas: são evidenciadas pelo contato do trabalhador com pacientes portadores de doenças infecciosas, infecto-contagiosas e parasitárias; pela manipulação de material contaminado e pela presença de insetos nocivos, além do grande número de microrganismos presentes em seu ambiente de trabalho;

- Cargas físicas: são aquelas a que os trabalhadores se expõem quando submetidos à condições inadequadas de iluminação, ventilação e umidade, à mudanças bruscas de temperatura, à vibração, ao ruído, a incêndios e choques elétricos e à radiação ionizante;

- Cargas químicas: são as decorrentes da manipulação de substâncias químicas (detergentes, desinfetantes, anestésicos, etc.), da manipulação de medicamentos, do contato com materiais de borracha (látex) e a fumaça do cigarro;

- Cargas mecânicas: são as que se evidenciam pelas quedas e agressões, além da manipulação de materiais pontiagudos e cortantes;

- Cargas fisiológicas: são aquelas a que os trabalhadores se expõem quando manipulam peso excessivo, trabalham em pé, assumem posições incômodas durante sua jornada de trabalho e quando são submetidos ao trabalho noturno e rodízio de turnos;

- Cargas psíquicas: são observadas quando os trabalhadores estão submetidos à atenção constante e supervisão estrita, à pressão da chefia e de outros profissionais, a horas extras e dobras de plantão, à falta de comunicação, à tensão, estresse e fadiga, à insatisfação, ao ritmo acelerado de trabalho, ao trabalho monótono e repetitivo e, ainda, a fatores como a falta de criatividade e autonomia e aqueles que levam ao abuso de álcoo e drogas, além da falta de articulação de defesas coletivas.

Independente da terminologia e da fundamentação teórica que a embase, as cargas ou os riscos ocupacionais do trabalho de enfermagem em Unidades Básicas de Saúde-UBS não têm sido abordados, o que nos estimulou a realizar este estudo. Portanto, nossa atenção está centrada no aprimoramento da compreensão sobre a percepção desses trabalhadores acerca dos riscos ocupacionais, a fim de melhor apreender a exposição desses trabalhadores nas UBS. Adotamos neste estudo o termo risco ocupacional por ser a terminologia mais conhecida pelos trabalhadores.

\section{OBJETIVOS}

- Captar a percepção dos trabalhadores de enfermagem de duas UBS sobre os riscos ocupacionais;

- Estabelecer um paralelo sobre os problemas de saúde relatados por esses trabalhadores como decorrentes da exposição a esses riscos.

\section{MÉTODO}

O estudo foi realizado em duas Unidades Básicas de Saúde (UBS-1 e UBS-2), caracterizadas como Centro de Saúde, cujo regime de atendimento não prevê internações. Estão localizadas na cidade de São Paulo, são instituições públicas, que têm como finalidades a assistência, ensino e pesquisa.

A população deste estudo foi composta por todos os trabalhadores de enfermagem presentes nas instituições durante o período de coleta de dados, perfazendo um total de 33 trabalhadores, sendo que 02 enfermeiros, 18 auxiliares de enfermagem, 05 visitadores sanitários e 01 atendente de enfermagem pertenciam à UBS- 1 (total de 26 trabalhadores) e 05 auxiliares de enfermagem e 02 visitadores sanitários, à UBS-2 (total de 7 trabalhadores). Deve-se destacar que os técnicos de enfermagem não participaram deste estudo, pois não estavam presentes nas instituições no período de coleta de dados e que os enfermeiros da UBS-2 também não participaram da coleta de dados.

Elegeu-se a entrevista como técnica de coleta de dados, utilizando-se para isso um instrumento elaborado especificamente para este estudo. Com este instrumento buscou-se caracterizar os trabalhadores de enfermagem em relação ao sexo, faixa etária e tempo 
de serviço; captar a percepção dos trabalhadores em relação aos riscos ocupacionais a que estão expostos no trabalho e a relação que estabelecem com os problemas de saúde. As entrevistas foram realizadas entre os meses de janeiro e fevereiro de 1999, de forma que o maior número possivel de trabalhadores estivessem presentes e pudessem colaborar com o estudo. A todos os trabalhadores foi solicitado o consentimento livre e esclarecido para participação no estudo, preservando todos os princípios éticos.

Os dados foram analisados de acordo a estatística descritiva, segundo as freqüências relativa e absoluta. Para a análise, foram consideradas a caracterização dos trabalhadores que constituíram a população deste estudo, a sua percepção acerca dos riscos ocupacionais a que estão expostos e os problemas de saúde por eles referidos.

\section{RESULTADOS E DISCUSSÃO}

\section{Resultados relativos à caracterização da população do estudo}

Tabela 01 - Distribuição dos trabalhadores de enfermagem da UBS-1 e UBS-2, segundo o sexo. São Paulo, 1999.

\begin{tabular}{l|cc|cc}
\hline \multicolumn{1}{c|}{ Sexo } & \multicolumn{2}{c|}{ U B S - 1 } & \multicolumn{2}{c}{ U B S -2 } \\
& N & \% & N & \% \\
\hline Feminino & 24 & 92,31 & 07 & 100,00 \\
Masculino & 02 & 7,69 & -- & -- \\
\hline Total & 26 & 100,00 & 07 & 100,00 \\
\hline
\end{tabular}

A população caracterizou-se como predominantemente feminina nas duas unidades, sendo que na UBS2 nenhum entrevistado era do sexo masculino, como pode ser visualizado na tabela 01 . Esta composição é condizente com a força de trabalho de enfermagem do País, que é constituída predominan-temente por mulheres.

Tabela 02 - Distribuição dos trabalhadores de enfermagem das UBS-1 e UBS-2, segundo a faixa etária (em anos). São Paulo, 1999.

\begin{tabular}{l|cc|cc}
\hline Faixa etária & $\mathbf{N}$ & $\begin{array}{c}\text { UBS-1 } \\
\mathbf{\%}\end{array}$ & $\mathbf{N}$ & $\begin{array}{c}\text { UBS-2 } \\
\%\end{array}$ \\
\hline 25 a 29 & 01 & 3,85 & - & - \\
30 a 34 & 03 & 11,52 & - & - \\
35 a 39 & 09 & 34,61 & 03 & 42,86 \\
40 a 44 & 07 & 26,92 & - & - \\
45 a 49 & 02 & 7,69 & - & - \\
50 a 54 & 03 & 11,52 & 01 & 14,29 \\
55 a 59 & 01 & 3,85 & 01 & 14,29 \\
60 e + & - & - & 01 & 14,29 \\
não respondeu & - & - & 01 & 14,29 \\
\hline Total & 26 & 100,00 & 07 & 100,00 \\
\hline
\end{tabular}

Quanto à faixa etária, observa-se na tabela 02 que mais de um terço dos trabalhadores de enfermagem das duas unidades têm entre 35 a 39 anos. Chama a atenção, a composição da força de trabalho das duas unidades, pois enquanto os trabalhadores da UBS-1 acima de 45 anos representam cerca de $77 \%$, na UBS2 eles constituem $42,87 \%$ do total da população estudada. No entanto, na UBS-1 existe uma maior distribuição nas faixas etárias mais jovens. Provavelmente, este fato relaciona-se ao tempo de existência dessas UBS, uma vez que o início de funcionamento da UBS-1 é mais recente que da UBS-2 e à inserção dos trabalhadores na unidade. Por outro lado, ambas são instituições públicas, o que garante ao servidor estabilidade no emprego, o que pode ter contribuído para a permanência dos trabalhadores da UBS-2 em seus cargos até idades mais elevadas.

Tabela 03 - Distribuição dos trabalhadores de enfermagem das UBS-1 e UBS-2, segundo o tempo de exercício profissional (em anos). São Paulo, 1999.

\begin{tabular}{l|cc|cc}
\hline $\begin{array}{l}\text { Tem po d e } \\
\text { exercicio } \\
\text { profissional }\end{array}$ & N & $\%$ & UBS- 1 & UBS-2 \\
\hline 01 a 04 anos & 01 & 3,85 & 02 & 28,57 \\
05 a 09 anos & 07 & 26,92 & -- & -- \\
10 a 19 anos & 16 & 53,85 & 03 & 42,86 \\
20 anos ou mais & 04 & 15,38 & 02 & 28,57 \\
\hline Total & 26 & 100,00 & 07 & 100,00 \\
\hline
\end{tabular}

Como é possivel verificar, $69,23 \%$ dos trabalhadores da UBS-1 e 71,43\% dos trabalhadores da UBS-2 exercem sua profissão há 10 anos ou mais. Isso significa que na duas unidades, cerca de $70 \%$ dos trabalhadores têm experiência no trabalho que realizam. Apenas uma pequena parcela da população tem um tempo de exercício profissional menor ou igual a 4 aros na UBS- 1 e na UBS-2, este percentual é maior, representam $28,57 \%$.

Comparando-se as tabelas 02 e 03, verifica-se que na UBS- 2 a porcentagem de trabalhadores com mais de 44 anos é maior e, também, a freqüência de trabalhadores com mais de 20 anos de exercício profissional, o indica que os trabalhadores tem significativa vivência profissional. 
Gráfico 01 - Distribuição dos trabalhadores de enfermagem das UBS-1 e UBS-2, segundo a categoria profissional. São Paulo, 1999.

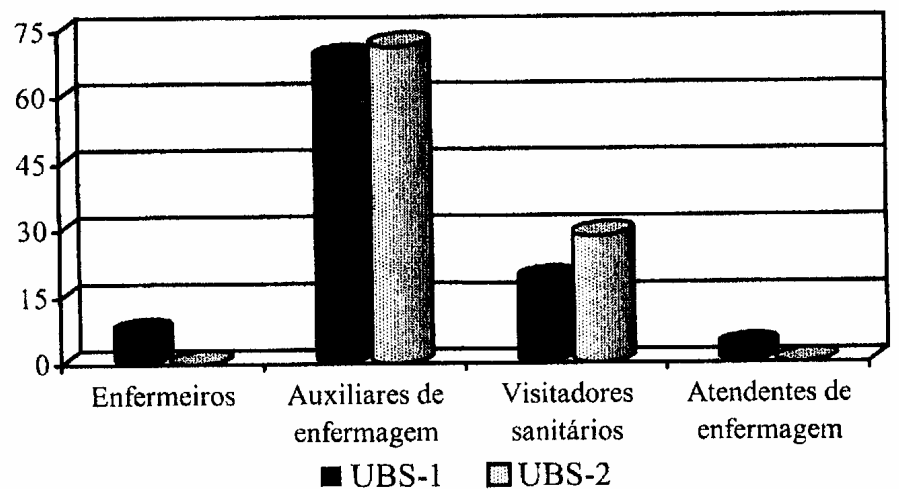

Em relação à categoria profissional, verifica-se no Gráfico 01, que 6,06\% dos trabalhadores da UBS1 são enfermeiros; uma vez que estes profissionais não participaram do estudo na UBS-2. Cerca de $70 \%$ são auxiliares de enfermagem, 20\% visitadores sanitários e 3\% são atendentes de enfermagem. Observa-se nesta constituição de força de trabalho, ainda, a participação do atendente, que contraria a lei do exercício profissional. Observa-se, também, uma pequena participação dos enfermeiros na equipe de trabalho.

\section{Resultados relativos à percepção dos trabalhadores acerca dos riscos ocupacionais e aos problemas de saúde}

A totalidade dos trabalhadores de enfermagem entrevistados considerou-se exposta a pelo menos um tipo de risco ocupacional. Lopes ${ }^{(3)}$ verificaram que $80,90 \%$ dos trabalhadores de enfermagem declararam a existência de riscos á saúde em seu ambiente de trabalho.

Esses trabalhadores evidenciaram em sua prática profissional exposição aos riscos:

- Biológicos - caracterizado pela transmissão de patógenos através das vias aéreas, e/ou secreções e sangue e através do contato direto com o cliente;
-Mecânicos - evidenciados pelos trabalhadores como os acidentes com objetos pérfuro-cortantes, quedas e agressão física;

-Psíquicos - apreendidos como o estresse, pressão, insatisfação com a situação da saúde pública (falta de medicamentos, campanhas de saúde pública ineficientes) e situações angustiantes (convivio com os problemas de saúde apresentados pelos clientes);

-Fisiológicos - apreendidos pelos trabalhadores como os agravos à coluna, danos ósteo-músculoarticulares, caminhadas excessivas durante a jornada de trabalho, necessidade de subir e descer escadas em demasia, execução de movimentos repetitivos, e a presença de mobílias inadequadas (que colaboram para a adoção de posturas incômodas);

-Riscos químicos - evidenciados pela exposição à medicações, vacinas e odores fortes; e aos riscos físicos, evidenciados através das queimaduras.

No Gráfico 02, é possivel observar os tipos de riscos ocupacionais aos quais os trabalhadores de enfermagem das UBS-1 e UBS-2 consideraram-se expostos.

Gráfico 02 - Freqüência (\%) de exposição aos riscos ocupacionais, segundo a percepção dos trabalhadores de enfermagem das UBS-1 e UBS-2. São Paulo, 1999.

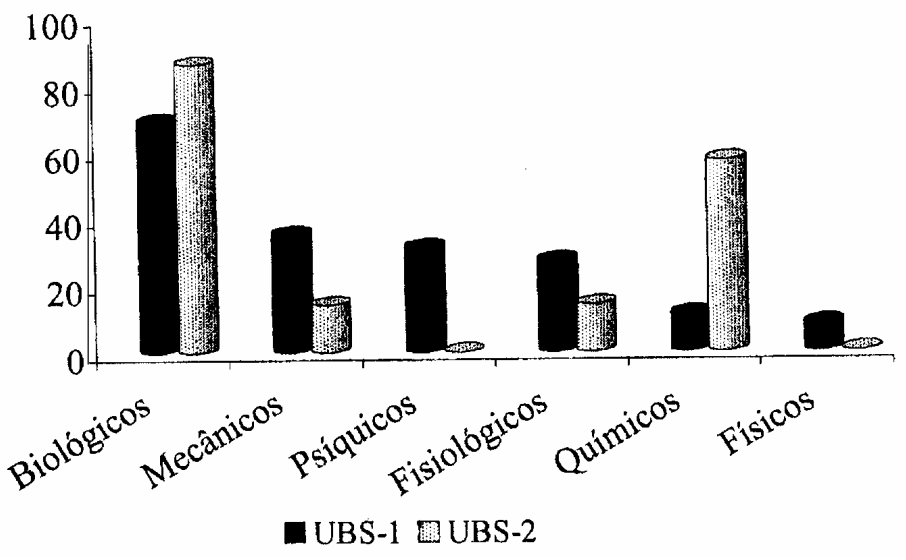


Verifica-se, no Gráfico 02, que na UBS-1 69\% dos trabalhadores de enfermagem consideraram-se expostos aos riscos biológicos; 35\% relataram exposição aos riscos mecânicos; a exposição aos riscos psíquicos foi evidenciada por \% dos trabalhadores; e $8 \%$ referiram exposição aos riscos físicos.

Em relação à UBS-2, observa-se que os trabalhadores de enfermagem não se consideraram expostos aos riscos psíquicos e físicos. Entretanto, $86 \%$ dos trabalhadores evidenciaram exposição aos riscos biológicos; $14 \%$, aos riscos mecânicos e aos riscos fisiológicos; e $57 \%$, aos riscos químicos. Esses achados podem estar relacionados às formas de organização e divisão do trabalho nesta instituição, bem como à utilização dos meios e instrumentos de trabalho, as quais possivelmente diferem daquelas encontradas na UBS-1.

Ainda, quanto à exposição aos riscos ocupacionais, 2 trabalhadores de enfermagem de cada uma das UBS consideraram-se expostos a todos os riscos, sem, no entanto, especificá-los.

Deste modo, depreende-se que os trabalhadores de enfermagem da UBS-2 consideraram estar mais expostos aos riscos biológicos e químicos que os trabalhadores da UBS-1; no entanto, estes observaram maior exposição aos riscos mecânicos, psíquicos, fisiológicos e físicos que os trabalhadores da UBS-2.

Em comparação à realidade de trabalho hospitalar, Silva(4) realizou estudo sobre os acidentes de trabalho entre os trabalhadores de enfermagem da Unidade de Centro de Material - UCM de sete hospitais da rede pública no Estado de São Paulo, no qual foram identificados todos os tipos de riscos ocupacionais, exceto os riscos psíquicos, assemelhando-se aos resultados encontrados para a UBS-2 no presente estudo.

Por outro lado, Silva(5) afirma que as morbidades psíquicas e as doenças psicossomáticas têm grande expressividade nas estatísticas brasileiras de auxiliodoença e, portanto, existe uma vinculação importante entre a Saúde do Trabalho e a Saúde Mental. Ressaltese, ainda, que na gênese dos riscos psíquicos encontrase a exposição simultânea a outros tipos de $\operatorname{riscos}^{(2)}$.

Os trabalhadores, sujeitos deste estudo, foram questionados sobre quais situações geravam maior exposição aos riscos ocupacionais. As situações por eles referidas foram agrupadas segundo o tipo de risco a que expunham os trabalhadores (tabela 04), de modo que fosse possível a comparação das mesmas com os tipos de riscos ocupacionais a que os trabalhadores haviam se considerado expostos.
Tabela 04 - Freqüência de exposição às situações geradoras de risco ocupacional, segundo a percepção dos trabalhadores de enfermagem das UBS-1 e UBS2. São Paulo, 1999.

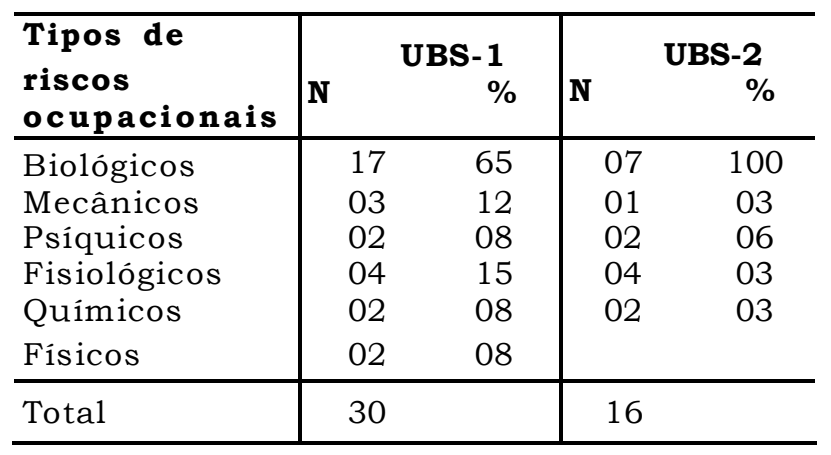

Apreende-se da tabela 04 que, com exceção das situações geradoras dos riscos biológicos, a identificação de exposição às situações geradoras dos diferentes tipos de riscos ocupacionais foi maior entre os trabalhadores da UBS-1. Provavelmente, tal fato é um indicativo de que os trabalhadores desta instituição estejam mais preparados, pois é a instituição que também conta com maior número de enfermeiros na força de trabalho, profissionais estes que, no papel gerencial, exercem a orientação da equipe de trabalho. Assim,. foram capazes de identificar mais situações geradoras de riscos ocupacionais do que os trabalhadores da UBS-2, que têm maior tempo de serviço e suposta experiência profissional.

Quanto aos riscos biológicos, os dados encontrados na UBS-1 são concordantes com aqueles anteriormente apresentados, uma vez que a frequência com que as situações geradoras deste tipo de risco é maior do que para as demais situações. Observa-se diferenças, em relação aos dados obtidos sobre a exposição aos riscos mecânicos, químicos e fisiológicos e as situações geradoras desses riscos na UBS- 1 .

Os trabalhadores da UBS-2 não referiram nenhuma situação que fosse geradora dos riscos físicos, o que está de acordo com os resultados apresentados, ou seja, esses trabalhadores não se consideraram expostos a tais riscos. Há concordância, também, no que se refere aos riscos biológicos, pois as situações geradoras desses riscos foram referidas pela totalidade dos trabalhadores de enfermagem da UBS-2. As situações geradoras de riscos psíquicos foram evidenciadas por $6 \%$ dos trabalhadores da UBS2. Tal fato chama a atenção, pois a exposição a estes riscos não havia sido identificada por estes trabalhadores. Por outro lado, os riscos químicos apresentaram um quadro inverso, uma vez que somente 3\% dos trabalhadores evidenciaram exposição às situações geradoras desses riscos, sendo que anteriormente $57 \%$ haviam relatado exposição a tais riscos. 
Esses resultados sugerem que tanto os trabalhadores da UBS-1 quanto aqueles da UBS-2 possuem dificuldades em reconhecer a gênese dos problemas de saúde decorrentes do trabalho. Tnão estabelecem, assim, a relação causa-efeito entre as situações vivenciadas por eles em seu ambiente de trabalho e a exposição aos riscos ocupacionais que essas situações proporcionam.

Tabela 05 - Freqüência de problemas de saúde referidos pelos trabalhadores de enfermagem das UBS1 e UBS-2, como decorrentes da exposição aos riscos ocupacionais. São Paulo, 1999.

\begin{tabular}{|c|c|c|c|c|}
\hline \multirow{2}{*}{ Problemas de saúde } & \multicolumn{2}{|c|}{ UBS-1 } & \multicolumn{2}{|c|}{ UBS-2 } \\
\hline & $\mathbf{N}$ & $\%$ & & $\%$ \\
\hline $\begin{array}{l}\text { Doenças contagiosas ou } \\
\text { infecto-contagiosas }\end{array}$ & 16 & 61 & 06 & 18 \\
\hline Ferimentos pérfuro-cortantes & 06 & 23 & 01 & 03 \\
\hline $\begin{array}{l}\text { Doenças do aparelho } \\
\text { osteo-musculo-articular }\end{array}$ & 04 & 15 & 01 & 03 \\
\hline Estresse & 05 & 19 & - & - \\
\hline Desgaste emocional & 05 & 19 & - & - \\
\hline Cansaço & 03 & 11 & - & - \\
\hline Distúrbio mental & 02 & 08 & - & - \\
\hline Hipertensão arterial & 01 & 04 & - & - \\
\hline $\begin{array}{l}\text { Queimaduras (vapor de } \\
\text { autoclave) }\end{array}$ & 01 & 04 & - & - \\
\hline Quedas & 01 & 04 & - & - \\
\hline $\begin{array}{l}\text { Diminuição da capacidade ou } \\
\text { limitação para o exercício de } \\
\text { determinadas funções }\end{array}$ & 01 & 04 & - & - \\
\hline TOTAL & 45 & & 08 & - \\
\hline
\end{tabular}

A tabela 05 mostra os problemas de saúde referidos pelos trabalhadores das UBS-1 e UBS-2, como decorrentes da exposição aos riscos ocupacionais.

Como é possivel verificar os 26 trabalhadores de enfermagem da UBS- 1 referiram 45 problemas de saúde, enquanto os sete da UBS-2 relataram apenas 08. Sendo assim, estes identificaram somente problemas de saúde que se relacionam com a exposição aos riscos biológicos $(18 \%)$, mecânicos $(3 \%)$ e psíquicos $(3 \%)$. Os problemas de saúde relacionados à exposição aos riscos químicos não foram identificados por nenhum dos trabalhadores.

Esses dados vêm reforçar a dificuldade dos trabalhadores em apreender as relações de causa-efeito entre as situações vivenciadas por eles em seu ambiente de trabalho, a exposição aos riscos ocupacionais que essas situações predispõem e os problemas de saúde gerados a partir dessa exposição.

As doenças infecciosas ou infecto-contagiosas apresentaram maior freqüência em ambas as UBS, sendo que $61 \%$ dos trabalhadores da UBS-1 e $18 \%$ dos trabalhadores da UBS-2 referiram que podem adoecer de alguma das morbidades que constituem essa categoria em decorrência da exposição aos riscos ocupacionais.

Diferentemente do que foi encontrado em estudos realizados em instituições hospitalares, os trabalhadores da UBS não referiram problemas respiratórios, enxaqueca e cefaléia, alergias e dermatites, intoxicação por substâncias quîmicas, problemas auditivos, problemas gastrintestinais, problemas de vias urinárias ou alcoolismo.

Em seu estudo sobre o desgaste de trabalhadores de enfermagem hospitalar, Gelbeck(6) verificou que os mesmos referiram o estresse e a fadiga, os problemas de coluna, a enxaqueca e a cefaléia, a infecção de vias aéreas superiores, as varizes, outros problemas ortopédicos, a gastrite, os problemas gênito-urinários, os problemas dermatológicos, a dor abdominal, a hipertensão arterial sistêmica, os acidentes de trabalho e as doenças infecciosas e parasitárias como sintomas ou doenças decorrentes da exposição ocupacional.

No hospital, também Silva(2) observou que os trabalhadores de enfermagem em seu estudo identificaram os seguintes sintomas ou doenças, em ordem decrescente de importância: ferimentos pérfurocortantes, doenças infecciosas, infecto-contagiosas e parasitárias; problemas respiratórios, desequilíbrio mental e desgaste emocional; enxaqueca e cefaléia; problemas de pele, problemas oculares e problemas auditivos; intoxicação por substâncias químicas, estresse, estafa e cansaço; distúrbios do sono; problemas gastrointestinais, dificuldade circulatória, varizes e dor nas pernas e irritabilidade; problemas de vias urinárias e hipertensão arterial; e doença psicossomática. Além desses, encontrou, ainda, problemas psiquiátricos e trauma emocional por pressão psicológica e ansiedade e o alcoolismo, os quais não tiveram uma ordem de classificação.

\section{CONCLUSÕES}

O presente estudo evidenciou a predominância do sexo feminino dentre os sujeitos, nas duas unidades; maior freqüência de trabalhadores nas faixas etárias acima dos 45 anos na UBS- 1 e abaixo desta idade na UBS-2, indicando uma inserção diferenciada dos trabalhadores nas duas unidades estudadas.

Em relação a percepção da exposição aos riscos ocupacionais, os trabalhadores de ambas UBS referiram, com freqüência maior que $60 \%$, a exposição 
aos riscos biológicos, sendo que pouco referiram os demais. Evidenciou-se, assim, que os trabalhadores das duas UBS possuem dificuldades em reconhecer a gênese dos riscos ocupacionais, ou seja, de estabelecer as relações de causa-efeito entre as situações vivenciadas no ambiente de trabalho, a exposição aos riscos ocupacionais que essas situações proporcionam e os problemas de saúde gerados a partir dessa exposição.

Mesmo assim, os tipos de riscos evidenciados foram semelhantes aos referidos para o trabalho em instituições hospitalares. No entanto, as freqüências observadas neste estudo, permitiram analisar que esta exposição percebida é diferenciada, tanto entre as UBS e as instituições hospitalares, quanto entre as duas UBS estudadas, principalmente no que se refere à percepção dos riscos biológicos em relação aos outros riscos.

A freqüência dos problemas de saúde referidos, relacionados à exposição a estes riscos, é condizente com a exposição aos riscos biológicos, mecânicos e fisiológicos, permitindo evidenciar a importância das doenças infecto-contagiosas, os ferimentos pérfurocortantes, o estresse e o desgaste emocional e as doenças do aparelho ósteo-músculo-articular compondo o perfil de morbidade destes trabalhadores. Estes dados permitem evidenciar a relação entre as atividades realizadas e o perfil saúde-doença dos trabalhadores.

Ao considerármos aos duas unidades de estudo, este perfil é semelhante ao evidenciado para os trabalhadores hospitalares. Diferenciam-se nas situações de exposição dos trabalhadores ao riscos na interação com a natureza do objeto de trabalho, os meios e instrumentos de trabalho e as formas de organização do trabalho. Desse modo, ao intencio-nármos a preservação da saúde do trabalhador, é imperativo estabelecer a relação entre a interação do trabalhador com esses elementos e a exposição às cargas de trabalho e a geração de problemas de saúde particulares.

\section{REFERÊNCIAS BIBLIOGRÁFICAS}

(1) Laurell AC, Noriega M. Processo de produção e saúde: trabalho e desgaste operário. São Paulo(SP): Hucitec; 1989.

(2) Silva VEF. O desgaste do trabalhador de enfermagem:relação trabalho de enfermagem e saúde do trabalhador. [Tese] São Paulo(SP): Escola de Enfermagem da Universidade de São Paulo; 1996.

(3) Lopes GT, Spindola T, Martins ERC. O adoecer em enfermagem segundo seus profissionais: estudos preliminares. Rev Enferm UERJ 1996; 4(1):9-18.

(4) Silva A. Trabalhador de enfermagem na unidade de centro de material e os acidentes de trabalho. [Tese] São Paulo(SP):Escola de Enfermagem da Universidade de São Paulo; 1996.

(5) Silva ES. Uma história de "crises de nervos": saúde mental e trabalho. In: Buschinelli JTP, Rocha E, Rigotto RM. organizador. Isto é trabalho de gente? vida, doença e trabalho no Brasil. São Paulo: Vozes; 1993. p. 609-35-

(6) Gelbcke FL. Processo saúde-doença e processo de trabalho: a visão dos trabalhadores de enfermagem de um hospital escola. [Dissertação] Rio de Janeiro(RJ): Escola de Enfermagem Alfredo Pinto da Universidade do Rio de Janeiro; 1991. 